\title{
Proinflammatory consequences of transgenic Fas ligand expression in the heart
}

\author{
David P. Nelson, ${ }^{1,2}$ Elizabeth Setser, ${ }^{1}$ D. Greg Hall, ${ }^{3}$ Steven M. Schwartz, ${ }^{1,2}$ \\ Timothy Hewitt, ${ }^{1}$ Raisa Klevitsky, ${ }^{1}$ Hanna Osinska, ${ }^{1}$ Don Bellgrau,, 4 \\ Richard C. Duke, ${ }^{4,5,6}$ and Jeffrey Robbins ${ }^{1}$
}

\begin{abstract}
${ }^{1}$ Division of Molecular Cardiovascular Biology, and
${ }^{2}$ Division of Cardiology, Department of Pediatrics, The Children's Hospital Research Foundation, Cincinnati, Ohio, USA

${ }^{3}$ Department of Pathology, College of Veterinary Medicine, University of Georgia, Athens, Georgia, USA

${ }^{4}$ Department of Immunology, University of Colorado School of Medicine, Denver, Colorado, USA

${ }^{5}$ Ceres Pharmaceuticals Ltd., Denver, Colorado, USA

${ }^{6}$ Department of Medicine, University of Colorado School of Medicine, Denver, Colorado, USA

Address correspondence to: Jeffrey Robbins, Division of Molecular Cardiovascular Biology,

The Children's Hospital Research Foundation, 3333 Burnet Avenue, Cincinnati, Ohio 45229, USA.

Phone: (513) 636-8098; Fax: (513) 636-5958; E-mail: robbj0@chmcc.org.
\end{abstract}

Received for publication August 19, 1999, and accepted in revised form March 20, 2000.

Expression of Fas ligand (FasL) renders certain tissues immune privileged, but its expression in other tissues can result in severe neutrophil infiltration and tissue destruction. The consequences of enforced FasL expression in striated muscle is particularly controversial. To create a stable reproducible pattern of cardiomyocyte-specific FasL expression, transgenic ( $\mathrm{Tg}$ ) mice were generated that express murine FasL specifically in the heart, where it is not normally expressed. Tg animals are healthy and indistinguishable from nontransgenic littermates. FasL expression in the heart does result in mild leukocyte infiltration, but despite coexpression of Fas and FasL in Tg hearts, neither myocardial tissue apoptosis nor necrosis accompanies the leukocyte infiltration. Instead of tissue destruction, FasL $\mathrm{Tg}$ hearts develop mild interstitial fibrosis, functional changes, and cardiac hypertrophy, with corresponding molecular changes in gene expression. Induced expression of the cytokines TNF- $\alpha$, IL-1 $\beta$, IL-6, and TGF- $\beta$ accompanies these proinflammatory changes. The histologic, functional, and molecular proinflammatory consequences of cardiac FasL expression are transgene-dose dependent. Thus, coexpression of Fas and FasL in the heart results in leukocyte infiltration and hypertrophy, but without the severe tissue destruction observed in other examples of FasL-directed proinflammation. The data suggest that the FasL expression level and other tissue-specific microenvironmental factors can modulate the proinflammatory consequences of FasL.

J. Clin. Invest. 105:1199-1208 (2000).

\section{Introduction}

Fas ligand (FasL, or CD95L) is a cell-surface molecule belonging to the TNF/nerve growth factor superfamily. FasL induces apoptotic cell death by binding to its membrane receptor Fas (also known as APO-1 or CD95) (1). Fas is ubiquitously expressed in various tissues, whereas FasL is expressed primarily in activated lymphocytes and in immune-privileged tissues such as the eye and testis. Interactions between Fas and FasL are integral to several immune processes, including regulation of lymphocyte homeostasis, T cell-mediated cytotoxicity, and immune privilege (1). Although interactions between Fas and FasL are vital to normal immune function, exaggerated or defective activity of the system can lead to significant pathology (2). Mice with mutations in Fas (lpr or $\left.l p{ }^{c} g\right)$ (3) or FasL (gld) (4) exhibit autoimmune/lymphoproliferative disorders due to disrupted T-cell homeostasis. Exaggerated FasL-based cytotoxicity can lead to a variety of autoimmune disorders, including fulminant hepatitis (5), graft-versus-host disease (6), diabetes (7-9), and Hashimoto's thyroiditis (10).
One of the most intriguing immune regulatory functions of FasL is its role in protecting certain tissues against immune-mediated injury, a phenomenon known as immune privilege (11-14). FasL expression in the eye (12) and testis (11) renders these tissues immune privileged, making them resistant to inflammatory injury and permissive to allograft survival. Likewise, FasL expression on some tumors confers tumor immunity (13-15). Constitutive FasL expression in these nonlymphoid tissues confers immune privilege by inducing apoptosis of Fas-expressing, infiltrating inflammatory cells.

Naturally occurring FasL-directed immune privilege has prompted investigation of the potential use of engineered FasL expression as an immunosuppressive agent for transplantation and other immune-mediated pathologic conditions. The resultant data conflict, fueling controversy over the consequences of enforced FasL expression in tissues where it is not normally expressed. Lau and colleagues (16) reported that FasLtransfected murine myoblasts protected pancreatic $\beta$ - 
a

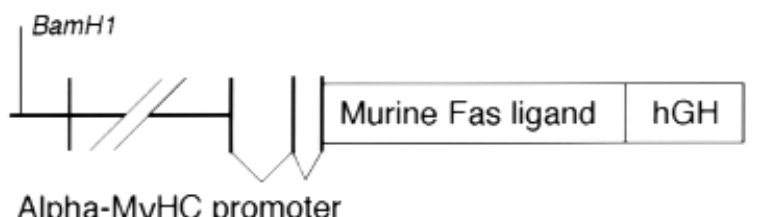

b

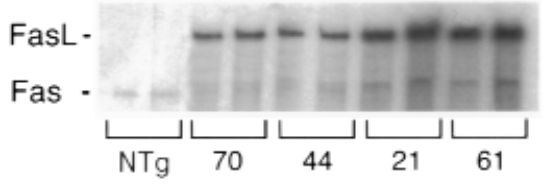

c

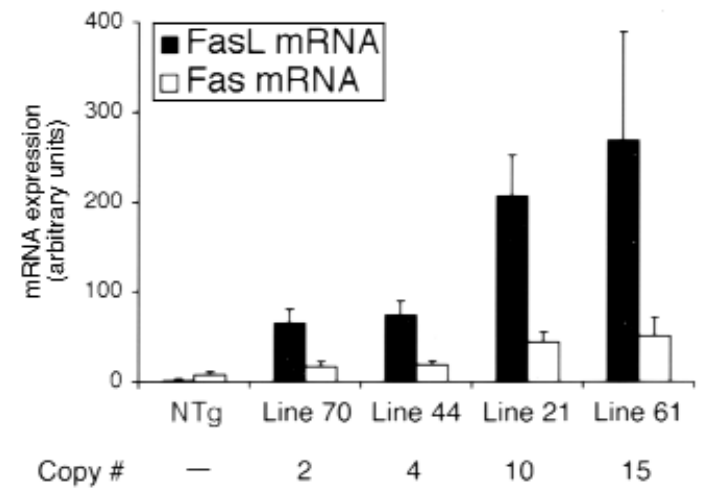

Figure 1

Murine FasL transgene construct and mRNA transcript analysis. (a) Murine FasL cDNA was isolated by RT-PCR, sequenced, ligated to the $\alpha$-MyHC promoter, and used to produce Tg animals (30). (b) Representative RNase protection assay showing that cardiac FasL expression corresponds to transgene copy number. (c) Total RNA was isolated from ventricles of NTg and Tg mice 8-12 weeks old with the transgene copy numbers indicated ( $n=6$ for each line). FasL and Fas transcripts were determined by multiplex RNase protection. Signal intensities were quantified on a Phosphorlmager and standardized by comparison to GAPDH signals. FasL expression is undetectable in NTg hearts, whereas steady-state FasL transcript levels correspond to the transgene copy number of each Tg line. Constitutive expression of Fas mRNA is detectable in NTg and $\mathrm{Tg}$ hearts. Fas and FasL transcripts are coexpressed in $\mathrm{Tg}$ hearts.

islet cells from transplant rejection when they were cotransplanted into the kidney capsule of allogeneic mice. Cotransplantation with nontransfected, FasLdeficient myoblasts resulted in prompt rejection of both myoblasts and islet cells. FasL-mediated immunosuppression has also been used successfully in animal models of arthritis (17), vascular injury (18), and renal (19) and hepatic (20) allograft transplantation. There is, however, increasing evidence that enforced expression of FasL can cause unwanted proinflammatory consequences, either by autologous apoptosis $(9,21)$ or by targeting the tissue for neutrophil infiltration and destruction (21-24). Induced expression of FasL in tumor cells $(21,22)$, islet cells $(23)$, and even myoblasts (24) can cause severe neutrophil infiltration and tissue destruction. FasL-mediated neutrophil infiltration requires functional Fas on host cells (presumably host neutrophils) $(23,24)$. Although the mechanisms resulting in these conflicting observations remain obscure, a variety of explanations have been proposed. Some investigators have suggested that Fas and FasL cannot be coexpressed in certain tissues $(21,24)$. It is possible that the consequences of enforced FasL expression are controlled by FasL expression levels (25), downstream cytokines $(2,7)$, tissue microenvironment factors (26), or the differential effects of membrane-bound and soluble forms of FasL $(27,28)$. Evaluation of the dose response of FasL expression has been complicated by difficulties in measuring and controlling expression levels of functional FasL in stable cell lines.

The effect of engineered FasL expression in striated muscle is particularly controversial $(16,24)$. To explore this process in detail, we created a stable, reproducible pattern of muscle cell-specific FasL expression. Using the $\alpha$-myosin heavy-chain promoter $(\alpha-\mathrm{MyHC}$ promoter) (29), we generated several lines of transgenic (Tg) mice that express FasL specifically in the heart, where it is normally absent. Analysis of these mice indicates that FasL expression in the heart is proinflammatory, resulting in mild leukocyte infiltration, interstitial fibrosis, and concentric hypertrophy. Strikingly, although both FasL and Fas are coexpressed in the hearts of these animals, FasL Tg hearts do not exhibit signs of cardiomyocyte apoptosis or necrosis, which differs markedly from the intense tissue destruction observed with enforced FasL expression in pancreatic islets, tumor cells, and myocytes. Furthermore, the molecular, histologic, and functional consequences of cardiac FasL expression are transgene-dose dependent, suggesting that the proinflammatory activity of FasL may depend on the extent of FasL tissue expression. These observations support the hypothesis that cellular and tissue-specific microenvironmental factors can modulate proinflammatory consequences of FasL.

\section{Methods}

Construction of $\mathrm{Tg}$ mice and copy number determination. Murine FasL cDNA was synthesized by RT-PCR using spleen RNA isolated from $\mathrm{FVB} / \mathrm{N}$ mice as a starting template. The PCR product was sequenced and then ligated to the $\alpha$-MyHC promoter (5,443 bp; GenBank accession no. U71441); the resultant construct (Figure 1a) was used to generate Tg mice (30). PCR screening identified founder animals, and four stable $\mathrm{Tg}$ lines (lines 21, 44, 61, and 70) were generated by breeding the founder mice with nontransgenic (NTg) animals. Subsequent offspring were screened by PCR. Transgene copy number was determined by Southern analysis using genomic DNA isolated from tail clips. DNA blots were analyzed using a random-primed full-length FasL cDNA as probe.

Determination of mRNA transcript expression. Total RNA was isolated from the ventricles of 8- to 12-week-old Tg and NTg mice. FasL, Fas, and cytokine mRNA expression was determined by multiplex RNase protection 
Table 1

Morphometric analysis

\begin{tabular}{|c|c|c|c|c|c|c|c|c|c|}
\hline & $\begin{array}{l}\text { BW } \\
(\mathrm{g})\end{array}$ & $\begin{array}{c}\text { Whole Ht } \\
\quad(\mathrm{mg})\end{array}$ & $\begin{array}{c}\text { RA } \\
(\mathrm{mg})\end{array}$ & $\begin{array}{l}\text { LA } \\
(\mathrm{mg})\end{array}$ & $\begin{array}{l}\mathrm{RV} \\
(\mathrm{mg})\end{array}$ & $\begin{array}{l}\text { LV } \\
(\mathrm{mg})\end{array}$ & $\begin{array}{l}\text { Lungs } \\
\text { (mg) }\end{array}$ & $\begin{array}{l}\text { Liver } \\
(\mathrm{mg})\end{array}$ & $\begin{array}{l}\text { Spleen } \\
(\mathrm{mg})\end{array}$ \\
\hline NTg & $21.1 \pm 2.4$ & $100 \pm 11$ & $5.9 \pm 1.6$ & $3.3 \pm 0.7$ & $16.3 \pm 4.1$ & $74.3 \pm 4.8$ & $139 \pm 10$ & $1,383 \pm 122$ & $99 \pm 8$ \\
\hline Line 70 & $23.4 \pm 0.9$ & $107 \pm 11$ & $4.6 \pm 0.9$ & $5.3 \pm 2.3$ & $23.1 \pm 5.0$ & $77.4 \pm 12.9$ & $132 \pm 5$ & $1,333 \pm 111$ & $91 \pm 3$ \\
\hline Line 21 & $21.5 \pm 2.0$ & $121 \pm 10^{\mathrm{A}}$ & $7.9 \pm 2.5^{\mathrm{A}}$ & $6.4 \pm 1.9^{\mathrm{A}}$ & $21.5 \pm 3.9$ & $85.4 \pm 5.0^{\mathrm{A}}$ & $133 \pm 6$ & $1,150 \pm 133$ & $119 \pm 19$ \\
\hline Line 61 & $23.2 \pm 3.5$ & $135 \pm 24^{\mathrm{A}}$ & $10.1 \pm 3.7^{\mathrm{A}}$ & $7.5 \pm 2.8^{\mathrm{A}}$ & $25.2 \pm 6.5$ & $92.3 \pm 11.7^{\mathrm{A}}$ & $115 \pm 19$ & $1,167 \pm 44$ & $106 \pm 6$ \\
\hline
\end{tabular}

according to the manufacturer's instructions (RiboQuant Multi-Probe RNase Protection Assay Systems; PharMingen, San Diego, California, USA). Transcript levels were measured in $6 \mathrm{NTg}$ hearts and 6 hearts from each $\mathrm{Tg}$ line. Hybridization signals were quantified on a PhosphorImager (Molecular Dynamics, Sunnyvale, California, USA). All steady-state transcript levels were normalized to GAPDH signal intensity to correct for loading.

Because cardiac hypertrophic changes and heart failure normally result in reactivation of the cardiac fetal genetic program, RNA blots were hybridized to transcript-specific probes for atrial natriuretic factor (ANF), $\alpha$ - and $\beta$-MyHC, cardiac and skeletal actin, Serca $2 \mathrm{a}$, and phospholamban (PLB) (30). Measurements were made in 8- to 12-week-old animals, $6 \mathrm{NTg}$ and 6 from each $\mathrm{Tg}$ line. To control for loading, all steady-state transcript levels were normalized to GAPDH signal intensity after correcting for background. A PhosphorImager was used to quantify hybridization signals.

Fas, FasL, and TGF- $\beta 1$ protein expression analysis. Fas and FasL protein expression was determined by Western analysis. Cardiac total protein lysates $(50 \mu \mathrm{g})$ from $\mathrm{NTg}$ and $\mathrm{Tg}$ hearts (from mice 8-12 weeks old) were separated by SDS-PAGE and transferred to a nitrocellulose membrane (Protran; Schleicher \& Schuell Inc., Keene, New Hampshire, USA). Protein samples from Fas-positive L1210 cells and FasL-positive K562 cells served as positive controls for Fas and FasL Western analyses, respectively. The protein blots were blocked for 3 hours at room temperature and then labeled with Fas- and FasL-specific antibodies overnight at $4{ }^{\circ} \mathrm{C}$ in $5 \%$ blocking solution (M-20 and Q-20; Santa Cruz Biotechnology Inc., Santa Cruz, California, USA). Bound primary antibody was detected by a rabbit Chemiluminescence Western Blotting Kit (Boehringer Mannheim Biochemicals Inc., Indianapolis, Indiana, USA). Parallel electrophoresis gels stained with Coomassie blue demonstrated consistent loading.

Myocardial TGF- $\beta 1$ protein expression was measured in protein lysates from 8- to 12-week-old NTg and Tg hearts using a commercial ELISA kit (R\&D Systems Inc., Minneapolis, Minnesota, USA). A standard curve was generated from concentration standards supplied with the kit.

Morphometric analysis. Hearts, lungs, liver, and spleen from 8- to 12-week-old NTg and Tg animals were weighed after dissecting away connective tissue. Atrial and ventricular cardiac chambers were separated and weighed. Five animals from each $\mathrm{Tg}$ line and $12 \mathrm{NTg}$ animals were analyzed.

Cardiomyocyte isolation and volume analysis. The volume of individual cardiomyocytes from 8- to 12-week-old Tg and NTg hearts was measured using a Z2 Coulter Channelyzer (Coulter Electronics Ltd., Miami, Florida, USA) (31). Mice were anticoagulated with heparin $(5,000$ $\mathrm{U} / \mathrm{kg}$ intraperitoneal) 30 minutes before anesthesia with metofane. Hearts were quickly removed and the aortas cannulated and connected to a Langendorff retrograde perfusion apparatus. The hearts were perfused with Joklik's Minimum Essential Medium (Sigma Biochemicals, St. Louis, Missouri, USA) with $34.5 \mathrm{mM} \mathrm{NaHCO}_{3}, 1$ $\mathrm{mM} \mathrm{MgSO}_{4}, 20 \mathrm{mM}$ 2,3-butanedione monoxime, and $0.2 \mathrm{mM}$ EGTA at pH 7.2 for 5 minutes. The perfusion medium was then changed to a similar medium containing $0.1 \%$ BSA and $150 \mathrm{U} / \mathrm{mL}$ type II collagenase (Worthington Biochemical Corp., Freehold, New Jersey, USA) instead of EGTA, for 8 minutes. The heart was removed from the perfusion apparatus, the atria were dissected off, and the remaining tissues were divided into right and left ventricles. The tissue was then minced, passed through $250-\mu \mathrm{m}$ nylon mesh, and fixed in $2.5 \%$ glutaraldehyde. Adequacy of digestion with retention of normal cellular architecture was verified in all samples by light microscopic analysis. Using a 200$\mu \mathrm{m}$ aperture calibrated with $42-\mu \mathrm{m}$-diameter microspheres (Coulter Electronics Ltd.), cell volume was measured in a sample of 2,500-5,500 cells. Measurements from individual animals were repeated to assure accuracy. The distribution of cell volumes was assessed using AccuComp software (Coulter Electronics Ltd.), and in all cases demonstrated a slightly skewed gaussian distribution. The mode was used as the best measure of central tendency. A shape factor of 1.43 was used to correct for the nonspherical shape of cardiomyocytes (31).

\section{Table 2}

Isolated cardiomyocyte cell volumes

\begin{tabular}{lcc} 
& \multicolumn{2}{c}{ Cardiomyocyte cell volume $\left(\mu \mathrm{m}^{3}\right)$} \\
& $\mathrm{RV}$ & $\mathrm{LV}$ \\
NTg & $30,181 \pm 2,035$ & $40,522 \pm 2,834$ \\
Line 61 & $32,631 \pm 3,108$ & $52,186 \pm 4,790^{\mathrm{A}}$ \\
& &
\end{tabular}




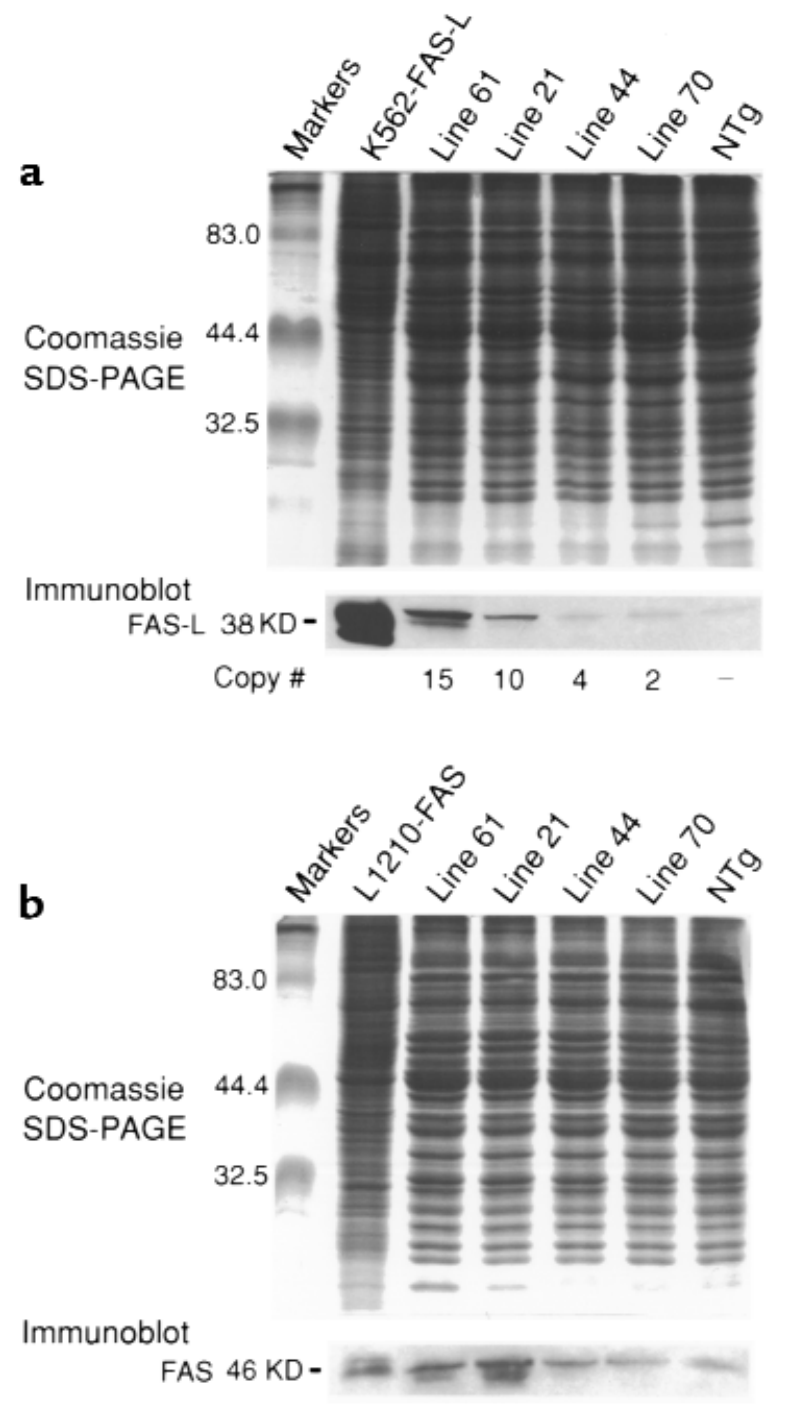

\section{Figure 2}

Fas and FasL protein are coexpressed in Tg hearts. Coomassie-stained gels (SDS-PAGE) and immunoblots showing increased levels of FasL (a) and Fas (b) protein in Tg hearts. Equivalent quantities of total cardiac protein lysates from NTg and Tg hearts from mice 8-12 weeks old were loaded and separated by electrophoresis. This was followed by either Coomassie brilliant blue dye staining or transfer to nitrocellulose for Western blot analysis. Protein lysates prepared from FasLtransfected K562 cells or Fas-transfected L1210 cells were loaded as positive controls. The immunoblot regions corresponding to the $M_{r}$ of FasL $(\sim 38 \mathrm{kDa})$ and Fas $(\sim 46 \mathrm{kDa})$ were labeled with anti-FasL or anti-Fas antibodies. Bound antibody was detected with labeled secondary antibody, and the signal was quantitated by Fluorimager (Amersham Pharmacia Biotech, Piscataway, New Jersey, USA) analysis. NTg hearts showed no FasL protein expression, whereas Tg hearts demonstrated protein expression corresponding to transgene copy number. Fas protein is expressed at low levels in NTg myocardium, and at increased levels in FasL-expressing $\mathrm{Tg}$ hearts.

The modes from $3 \mathrm{Tg}$ hearts (line 61) and 3 NTg hearts were averaged and compared.

Immunocytochemistry. Cardiomyocytes from hearts of mice $8-12$ weeks old were isolated as described above for cell-volume measurements, except that they were fixed in $4 \%$ paraformaldehyde rather than in glutaraldehyde. Cells were dried on glass slides and treated with $1 \%$ Triton X-100 for 30 minutes, and blocked in $0.5 \%$ BSA for 1 hour. Cells were labeled with antiFasL antibody (N-20; Santa Cruz Biotechnology Inc.) for 1 hour at room temperature. They were then labeled with FITC-conjugated donkey anti-rabbit IgG secondary antibody (Jackson ImmunoResearch Laboratories, West Grove, Pennsylvania, USA) and propidium iodide for 1 hour at room temperature. Labeled cells were visualized by confocal microscopy (CLSM2010; Molecular Dynamics).

Histopathology and immunohistochemistry. Tg and $\mathrm{NTg}$ animals (8-12 weeks old) were anesthetized and the hearts were perfused with relaxation buffer $(25 \mathrm{mM}$ $\mathrm{KCl}$ and 5\% dextrose in PBS) with heparin to wash out blood. The hearts were removed, fixed in $10 \%$ formalin, and embedded in paraffin. Paraffin-embedded myocardial sections $(5 \mu \mathrm{m})$ stained with hematoxylin and eosin or Gomori's one-step trichrome stain were examined by light microscopy. To demonstrate neutrophils within the myocardium of Tg hearts, paraffin-embedded myocardial sections $(5 \mu \mathrm{m})$ from NTg and line 61 mice were treated with Leder stain (fuchsin acid, sodium nitrite, and naphthol-ASD chloroacetate esterase in PBS), which identifies chloroacetate esterase within neutrophils, and examined by light microscopy.

TUNEL staining and DNA ladder analysis. Evidence of apoptosis or necrosis was assessed by detection of nuclear DNA fragmentation in paraffin-fixed myocardial sections by terminal deoxynucleotidyl transferase-mediated dUTP nick end-labeling (CardioTACS Blue TUNEL staining; Trevigen Inc., Gaithersburg, Maryland, USA) according to the manufacturer's instructions. TUNEL-stained myocardial sections were examined by light microscopy. The presence of apoptotic cells in NTg and Tg hearts was also assessed by genomic DNA agarose gel electrophoresis. Using a mortar and pestle under liquid nitrogen, myocardial tissue from $\mathrm{Tg}$ and $\mathrm{NTg}$ hearts from mice 8-12 weeks old was finely ground and then digested overnight in homogenization buffer $(10 \mathrm{mM}$ Tris, $100 \mathrm{mM} \mathrm{NaCl}, 25 \mathrm{mM}$ EDTA, $0.5 \% \mathrm{SDS}, 1 \mathrm{mg} / \mathrm{mL}$ proteinase K; $\mathrm{pH}$ 8.0). Protein was precipitated with $2.4 \mathrm{M} \mathrm{NaCl}$ and removed by centrifugation at $10,000 \mathrm{~g}$ for 30 minutes. Supernatants were extracted with phenol and chloroform. DNA was precipitated with isopropanol, treated with RNase A for 30 minutes at $37^{\circ} \mathrm{C}$, and precipitated again with isopropanol. Isolated DNA $(10 \mu \mathrm{g})$ was size separated in $1.4 \%$ agarose gels and visualized with ethidium bromide. Genomic DNA isolated from staurosporine-treated apoptotic neonatal rat cardiomyocytes served as positive control for apoptosis and DNA laddering.

Transmission electron microscopy. Hearts from NTg and Tg mice 8-12 weeks old were fixed by perfusion with a glutaraldehyde-containing cardioplegic solution. The heart was dissected into six sections: left and right atrial flaps, left and right ventricular free wall, septum, and apex. These specimens were postfixed in osmium tetrox- 

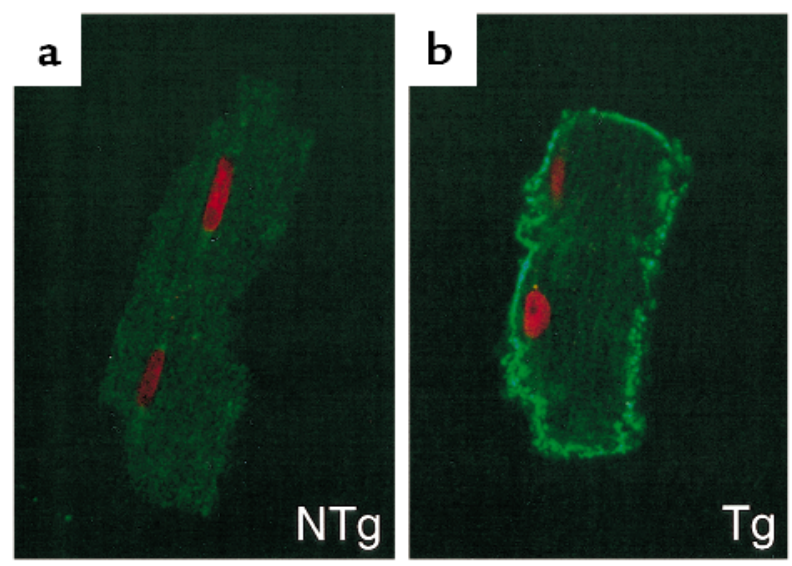

Figure 3

Immunocytochemistry of NTg and Tg isolated cardiomyocytes. (a) Isolated cardiomyocyte from an NTg mouse with minimal background fluorescence. Nuclei appear in red (propidium iodide staining). (b) Dual-nucleated cardiomyocyte from $\mathrm{Tg}$ heart (line 61, high expression) demonstrating abundant membrane-bound FasL protein.

ide and dehydrated, and then embedded in Poly/Bed 812 embedding media (Polysciences Inc., Warrington, Pennsylvania, USA). Random blocks were sectioned, counterstained, and examined using a Zeiss Omega 912 transmission electron microscope at an accelerating voltage of $100 \mathrm{kV}$.

Functional analysis of $\mathrm{Tg}$ and NTg hearts. To determine whether cardiac FasL expression alters contractile function, we assessed physiologically relevant parameters of cardiovascular function in 12-week-old sex-matched $\mathrm{NTg}$ and $\mathrm{Tg}$ hearts using the isolated work-performing heart preparation (30). Cardiac minute work was varied from 250 to $750 \mathrm{mmHg} \times \mathrm{mL} / \mathrm{min}$ by increasing cardiac venous return. Function was assessed in hearts with both low (line 70) and high levels (line 61) of FasL expression (see Table 3).

Statistical analysis. Comparisons between NTg hearts and the four FasL Tg heart lines were tested by ANOVA. Differences between specific groups were assessed by unpaired $t$ test with Bonferroni correction. A $P$ value of less than 0.05 was considered statistically significant.

\section{Results}

FasL expression in $\mathrm{Tg}$ hearts is dependent on transgene copy number. Using the $\alpha-M y H C / F a s L$ transgene construct (Figure 1a), four Tg lines of animals that exhibited germ line transmission were generated. The Tg lines demonstrated a range of transgene copy numbers by Southern analysis $(15,10,4$, and 2 copies in lines 61 , 21,70 , and 44 , respectively).

To determine levels of FasL expression in Tg hearts, FasL transcripts were assessed by RNase protection (Figure $1, \mathrm{~b}$ and $\mathrm{c}$ ), and protein expression was determined by Western analysis (Figure 2a). FasL mRNA and protein expression is undetectable in NTg hearts, whereas steady-state FasL transcript and protein expression correspond to the transgene copy number of each $\mathrm{Tg}$ line. Protein lysates from FasL-transfected K562 cells served as positive controls for Western blots. It has been suggested that coexpression of FasL with Fas may lead to neutrophil infiltration and tissue destruction in myoblasts (25) and $\beta$-islet cells $(7,8,23,24)$. Fas mRNA and protein are expressed at low levels in NTg myocardium, and at increased levels in Tg hearts (Figures $1 \mathrm{~b}$ and $2 \mathrm{~b}$ ), thus demonstrating coexpression of Fas and FasL in Tg hearts. Immunostaining of isolated cardiomyocytes (Figure 3) shows that FasL is undetectable on NTg cardiomyocytes, whereas $\mathrm{Tg}$ cardiomyocytes show the diffuse immunoreactivity of membrane-bound FasL.

Cardiac FasL expression is proinflammatory without accompanying cardiomyocyte destruction. All lines of FasL $\mathrm{Tg}$ mice appear overtly healthy and show no signs of distress during normal activity. Postmortem examination of all organs revealed pathologic changes in the heart only. Analysis of Tg hearts with low-level FasL expression (lines 44 and 70 ) revealed no obvious morphologic or histologic changes. The Tg hearts expressing higher levels of FasL (lines 21 and 61) appear globoid, with mild concentric hypertrophy involving atria and ventricles (an example from line 61 is shown in Figure 4). Histologic analyses of these hearts demonstrated diffuse concentric hypertrophy and mild interstitial changes consisting of leukocyte infiltration, interstitial hypercellularity, and fibrosis (Figure 4, b, d, and f). The interstitial hypercellularity consists of fibroblasts, macrophages, and leukocytes. Leder-stained myocardial sections from 8 to 12 -weekold line $61 \mathrm{Tg}$ hearts demonstrate that the infiltrating leukocytes are predominantly neutrophils (Figure 4h).

Despite leukocyte infiltration, the reticular architecture of the heart is preserved, and cardiomyocytes appear intact with no signs of myocardial tissue injury or cardiomyocyte dropout. Evidence of apoptotic or necrotic cell destruction was assessed in myocardial sec-

Table 3

Working heart ventricular function analysis

\begin{tabular}{|c|c|c|c|c|c|}
\hline & NTg & Line 70 & $\%$ Change & Line 61 & $\%$ Change \\
\hline Working heart & $(n=13)$ & $(n=6)$ & & $(n=7)$ & \\
\hline $\begin{array}{l}\text { Heart rate }(\text { beats } / \mathrm{min}) \\
+\mathrm{d} P / \mathrm{d} t(\mathrm{mmHg} / \mathrm{msec}) \\
-\mathrm{d} P / \mathrm{d} t(\mathrm{mmHg} / \mathrm{msec})\end{array}$ & $\begin{array}{c}355 \pm 2 \\
6,523 \pm 140 \\
4,636 \pm 100\end{array}$ & $\begin{array}{c}358 \pm 7 \\
6,575 \pm 178 \\
4,445 \pm 110\end{array}$ & $\begin{array}{l}\text { NS } \\
\text { NS } \\
\text { NS }\end{array}$ & $\begin{array}{c}386 \pm 4 \\
7,027 \pm 143 \\
3,158 \pm 55\end{array}$ & $\begin{array}{r}+9^{\mathrm{B}} \\
+8^{\mathrm{A}} \\
-32^{\mathrm{B}}\end{array}$ \\
\hline
\end{tabular}

${ }^{A} P<0.05,{ }^{B} P>0.001, T g$ vs. NTg, unpaired Student's $t$ test; NS, not significant. 
tions by detection of nuclear DNA fragmentation using TUNEL staining. Detailed examination of myocardial sections revealed no sign of cardiomyocyte necrosis or apoptosis in either NTg or FasL Tg hearts. The only TUNEL-positive cells observed in $\mathrm{Tg}$ hearts were occasional interstitial leukocytes (Figure 5b). Diffuse TUNEL staining is clearly visible in the stained sections from DNase-treated NTg myocardium (Figure 5a, inset). These results were confirmed by the absence of DNA ladders on agarose gel electrophoresis of DNA isolated from FasL Tg hearts with low (line 70) and high levels (line 61) of FasL expression (Figure 5c).

Ultrastructural analysis of line 61 hearts (Figure 6) consistently showed marked interstitial changes with type I collagen deposition and leukocyte infiltration (predominately neutrophils). The sarcomeric organization of $\mathrm{Tg}$ myocardium was unaffected, however, and there were no ultrastructural signs of tissue destruction, cardiomyocyte apoptosis, or necrosis. Only infiltrating leukocytes demonstrated morphologic changes characteristic of apoptosis, with clumped chromatin and cytoplasmic shrinkage. Apoptotic leukocytes engulfed by phagocytes were seen.

Other investigators have proposed that proinflammatory consequences of FasL in other tissues may be mediated or modulated by various cytokines $(2,21,26)$. To determine whether cytokine expression is induced in FasL Tg hearts, mRNA transcripts for potential candidate cytokines (TNF- $\alpha$, IL- $1 \beta$, IL- 6 , and TGF- $\beta$ ) were assessed by multiplex RNase protection. FasL Tg hearts demonstrated increases in transcript levels of all cytokines analyzed in a transgene dose-dependent manner (Figure 7a). Because TGF- $\beta 1$ has been shown to modulate the proinflammatory effects of FasL (26), myocardial TGF- $\beta 1$ protein concentration was assayed by ELISA. TGF- $\beta 1$ protein levels were increased in Tg hearts compared with NTg hearts, and were comparable to increases measured in TGF- $\beta 1$ mRNA expression (Figure 7b).

Myocardial FasL expression results in transgene dose-dependent hypertrophy with characteristic molecular changes in gene expression. The histologic and ultrastructural analysis of FasL Tg hearts implied that coexpression of Fas and FasL in the heart resulted in mild hypertrophy, rather than myocardial tissue destruction. To further investigate the apparent hypertrophic phenotype, morphometric analysis of 8- to 12-week-old $\mathrm{NTg}$ and Tg hearts was performed. Results demonstrated increased whole-heart and chamber weights in lines 21 and $61(P<0.01$ for both absolute and indexed chamber weights), but no morphometric changes in lines 44 and 70 (Table 1). There were no differences in lung, liver, or spleen weights among NTg and Tg animals.
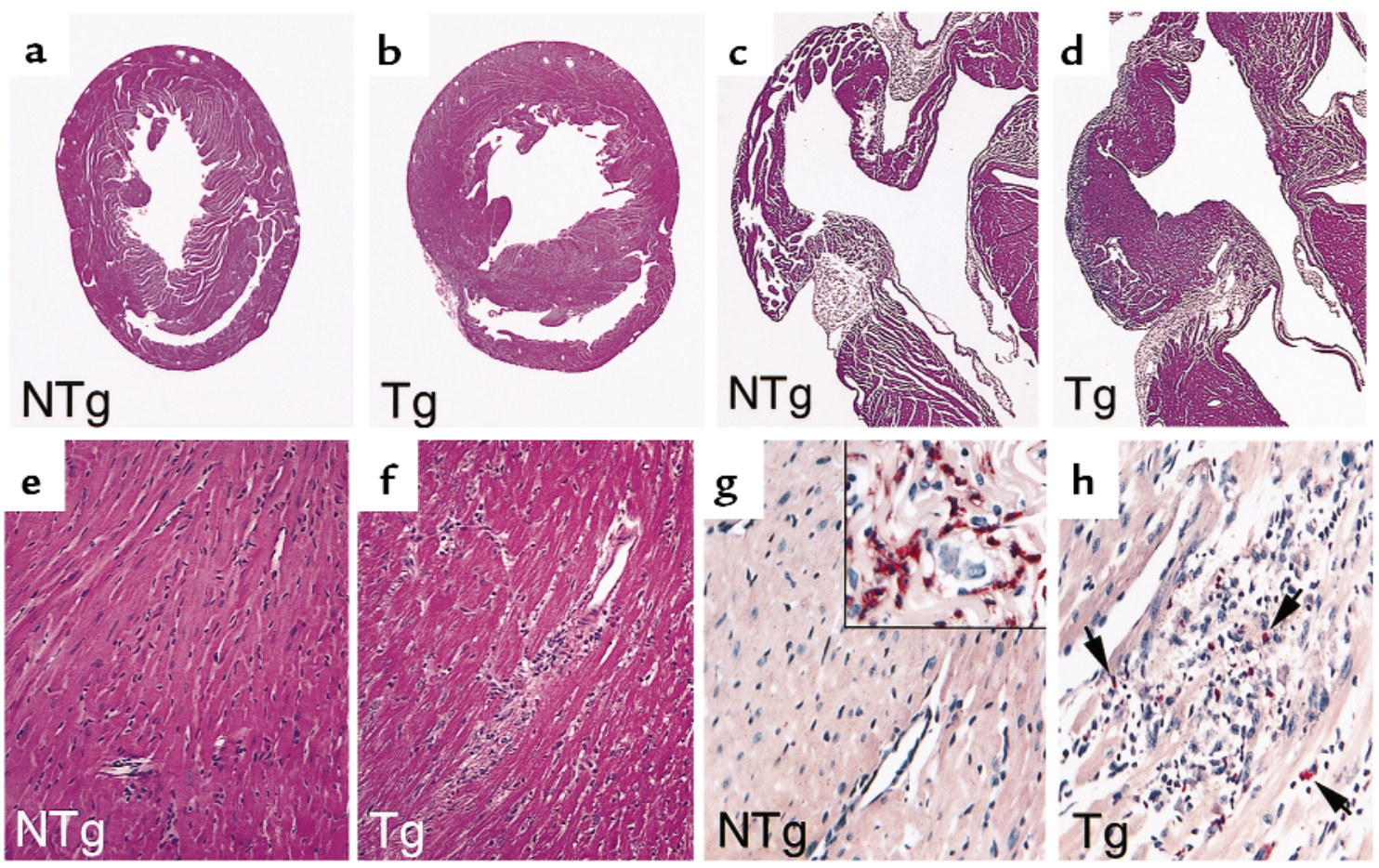

\section{Figure 4}

Histopathologic changes in FasL Tg hearts. Transverse (a, b), atrial (c, d), and ventricular (e, f) sections from representative 12-week-old NTg and FasL line $61 \mathrm{Tg}$ animals, stained with hematoxylin and eosin. Tg hearts with low-level FasL expression (lines 44 and 70 ) are normal (not shown). Shown is a representative line 61 heart (high-level FasL expression) demonstrating atrial and ventricular concentric hypertrophy $(\mathbf{b}, \mathbf{d})$, and interstitial hypercellularity with fibrosis and mild leukocyte infiltration $(\mathbf{d}, \mathbf{f})$. There is no evidence of cardiomyocyte necrosis, apoptosis, or dropout, but occasional interstitial leukocytes demonstrate dense fragmented nuclei consistent with apoptosis. The infiltrating leukocytes are predominantly neutrophils, as demonstrated by ventricular sections from NTg (g) and line 61 FasL Tg hearts (h) treated with Leder stain, which specifically detects chloroacetate esterase in neutrophils. The inset ing displays Leder-stained inflamed human appendix used as a positive control. 


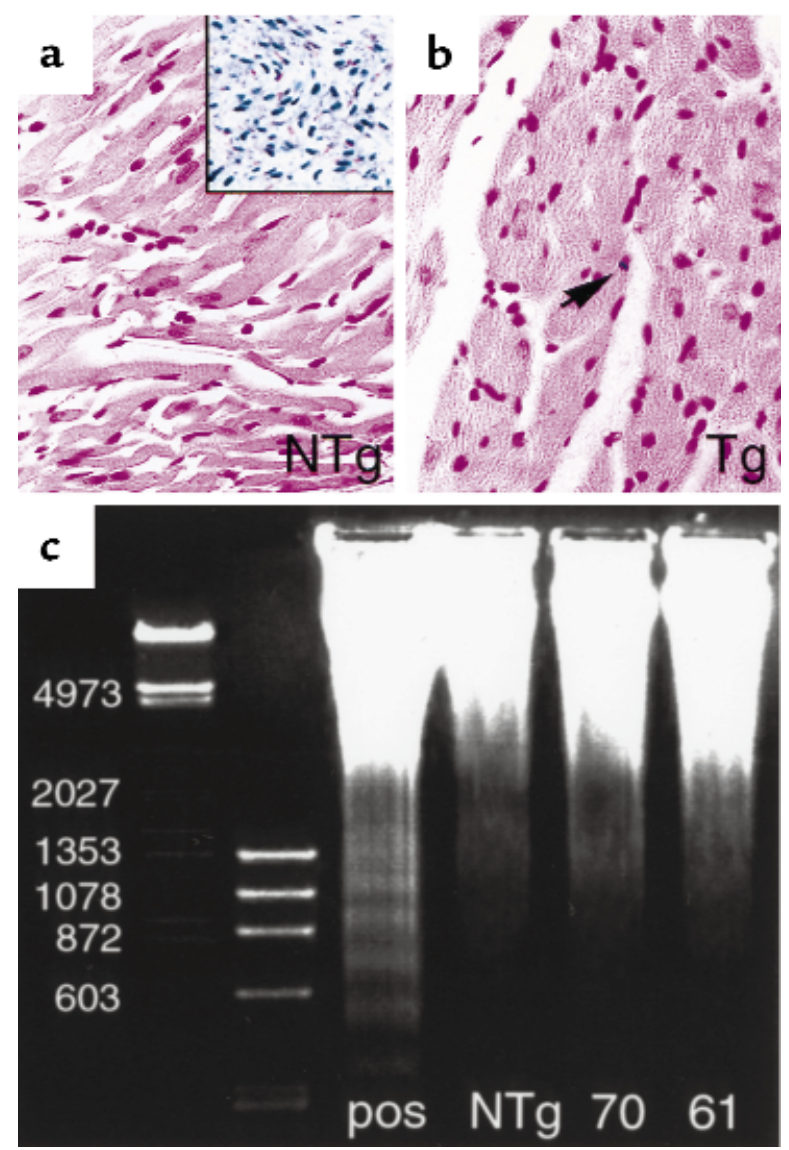

\section{Figure 5}

Cardiomyocyte apoptosis was not detected in 12-week-old FasL Tg hearts by TUNEL or DNA ladder analysis. Paraffin sections of NTg (a) and line $61 \mathrm{FasL} \operatorname{Tg}(\mathbf{b})$ ventricle were stained for apoptotic cells through the labeling of nuclear DNA fragments. The inset in a displays DNasetreated NTg myocardium as a positive control for the TUNEL stain. TUNEL analysis demonstrates no apoptotic or necrotic cardiomyocytes in either NTg or Tg myocardium. Small numbers of TUNEL-positive leukocyte nuclei (arrows) are seen in the interstitium of Tg hearts. The scattered leukocytes, predominantly neutrophils and lymphocytes, are presumably apoptotic. DNA laddering was not detected in NTg, line 70, or line 61 Fas $\mathrm{L}$ Tg hearts $(\mathbf{c})$. Staurosporine-treated rat cardiomyocytes served as positive control for DNA ladder analysis.

$\mathrm{Tg}$ and NTg cardiomyocyte cell volumes were measured with a Coulter Channelyzer (31). The Tg right ventricular cardiomyocyte cell volumes (line 61) tended to be greater than those in NTg hearts but did not reach statistical significance, whereas $\mathrm{Tg}$ left ventricular myocytes were approximately $20 \%$ larger than those from NTg hearts $(P=0.04$; Table 2$)$. These data indicate that the cardiac enlargement observed in FasL-expressing hearts is, at least in part, due to true cardiomyocyte hypertrophy.

To determine whether the hypertrophic changes correspond to molecular changes in gene expression, NTg and FasL Tg hearts were analyzed for mRNA changes characteristic of hypertrophy. Because cardiac hypertrophy can result in reactivation of the cardiac fetal genetic program and downregulation of PLB and Serca 2a, mRNA transcript levels for ANF, $\alpha$ - and $\beta$-MyHC, cardiac and skeletal actin, Serca 2a, and PLB were assessed. FasL Tg hearts showed transgene dosedependent changes in gene expression (Figure 8). Hearts from lines 21 and 61 consistently showed changes in gene expression characteristic of hypertrophy (increases in ANF, $\beta$-MyHC, and skeletal actin; decreases in $\alpha-\mathrm{MyHC}$ and cardiac actin). The increases in ANF were particularly significant. Although hearts with lower FasL expression show no phenotypic changes (lines 44 and 70), they do demonstrate decreased expression of PLB and Serca 2a transcripts. Thus, FasL-mediated hypertrophy is accompanied by characteristic molecular changes in gene expression in a transgene dose-dependent manner.

The proinflammatory effects of cardiac FasL expression have mild functional consequences. To determine whether the phenotypic changes associated with cardiac FasL expression alter myocardial function, working heart analyses compared systolic and diastolic functional parameters in 12-week-old NTg and Tg hearts with low and high levels of FasL expression (Table 3). FasL $\mathrm{Tg}$ hearts with low-level FasL expression (line 70), which demonstrate no histologic changes and minimal molecular changes, showed no significant differences in functional parameters. In contrast, line 61 hearts demonstrated a $35 \%$ decrease in maximal rate of relaxation $(-\mathrm{d} P / \mathrm{d} t)$, and slight increases in maxi-
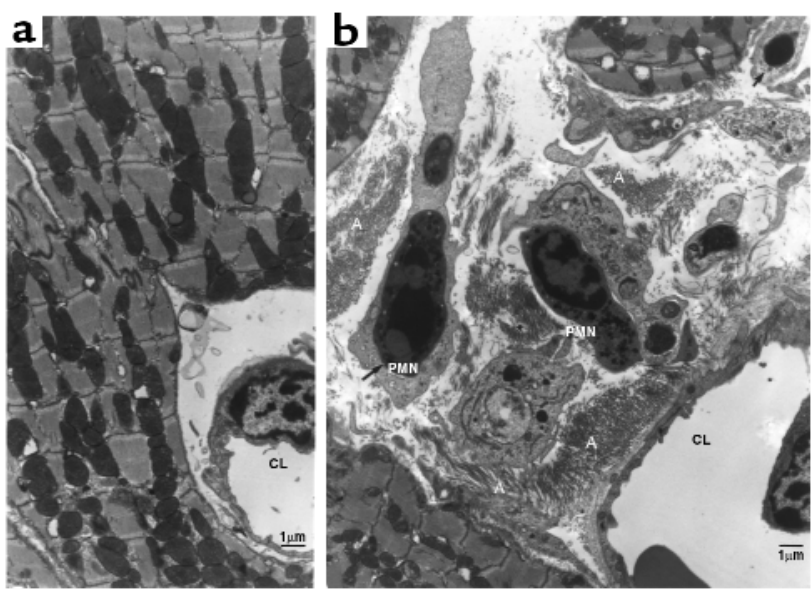

\section{Figure 6}

Ultrastructural analysis revealed interstitial changes in line 21 and line 61 FasL Tg hearts, whereas hearts from line 44 and line 70 animals were normal. Multiple animals derived from each line were studied; shown are representative transmission electron microscopy sections from 12-week-old NTg (a) and line $61 \mathrm{Tg}$ (b) left ventricle $(\times 8,800)$. Normal ultrastructural features of NTg myocardium (a) include thinwalled capillary lumen $(\mathrm{CL})$, minimal interstitial collagen deposition, and well-organized cardiomyocyte sarcomas. By contrast, line $61 \mathrm{Tg}$ myocardium (b) demonstrates marked interstitial changes with type I collagen deposition $\left({ }^{A}\right)$ and leukocyte infiltration (predominantly lymphocytes and neutrophils [PMN]). Many interstitial leukocytes demonstrate morphologic changes characteristic of apoptosis, with clumped chromatin and cytoplasmic shrinkage (arrows). Apoptotic leukocytes are frequently shown engulfed by phagocytes. The sarcomeric organization of FasL Tg myocardium is unaffected, with no indication of cardiomyocyte apoptosis or necrosis. 

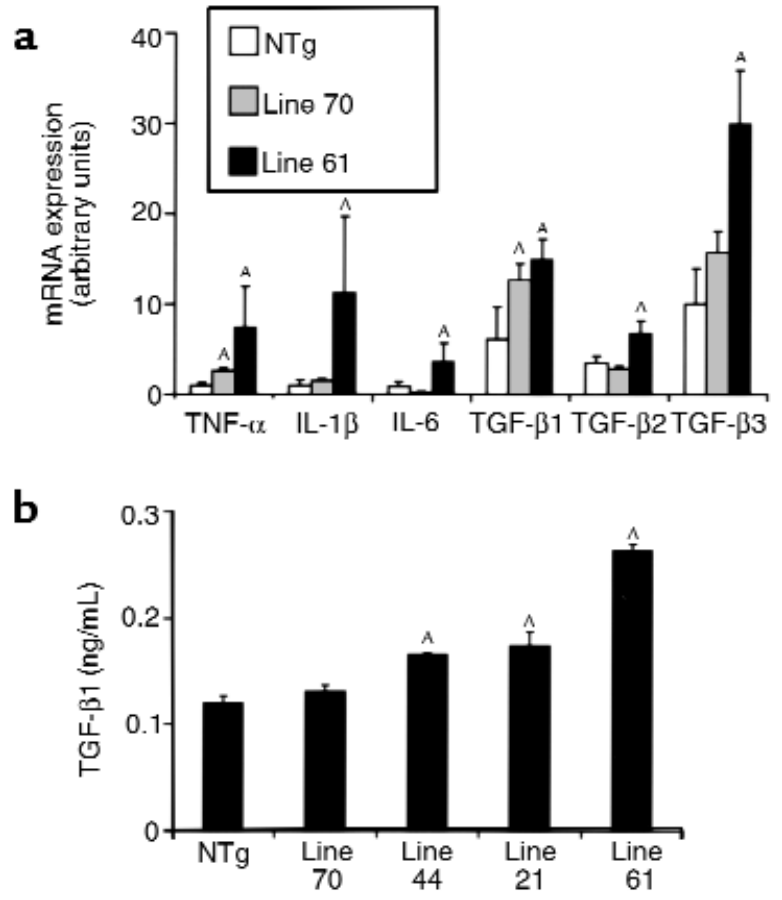

Figure 7

FasL $T g$ hearts demonstrate transgene dose-dependent changes in cytokine expression. (a) Transcript expression levels for TNF- $\alpha$, IL-1 $\beta$, IL-6, and TGF- $\beta$ were determined by multiplex RNase protection assay in NTg and FasL Tg hearts from mice 8-12 weeks old. Shown are data from six NTg and six Tg hearts from lines 70 and 61. Compared with NTg controls, FasL Tg hearts demonstrate increased expression for all cytokines displayed ( ${ }^{A} P<0.05$ ). (b) Cytokine expression in Fas $L \mathrm{Tg}$ hearts corresponds to transgene copy number. Myocardial TGF- $\beta 1$ concentration in NTg and Tg hearts was evaluated by ELISA. TGF- $\beta 1$ levels were increased in Tg hearts $\left({ }^{A} P<0.05\right)$.

mal rate of contractility $(+\mathrm{d} P / \mathrm{d} t)$ and heart rate relative to NTg hearts. Alterations in diastolic function could result from increased chamber stiffness (due to interstitial fibrosis). Alternatively, altered calcium handling caused by downregulation of Serca 2a and PLB could have functional effects; it is intriguing that decreases in measured $-\mathrm{d} P / \mathrm{d} t$ parallel the decreases observed in Serca 2a and PLB transcripts (Figure 8, Table 3).

\section{Discussion}

Interactions between Fas and FasL are remarkably complex (2). Whereas FasL expression confers immune privilege to some tissues, it can also lead to neutrophil infiltration and tissue destruction. The model described above indicates that cardiomyocytespecific FasL expression is proinflammatory, resulting in mild leukocyte infiltration. However, in sharp contrast to other examples of FasL-mediated inflammation (21-24), the leukocytic infiltrate was not accompanied by myocardial tissue necrosis or apoptosis. Instead of tissue destruction, FasL Tg hearts develop mild interstitial fibrosis and cardiomyocyte hypertrophy, with corresponding molecular changes in cardiac gene expression. The magnitude of these changes is dependent upon FasL expression, which in turn corresponds to transgene copy number.

Proinflammatory changes in FasL Tg animals are limited to the heart. Postmortem analysis of FasL Tg animals demonstrated no pathologic changes in other organs, including thymus, spleen, liver, and lymph nodes. Furthermore, subgroup FACS ${ }^{\circledR}$ analysis of lymphocytes isolated from lymph nodes, spleen, and thymus revealed no overt differences between NTg and $\mathrm{Tg}$ animals (data not shown). Takeuchi et al. have also generated FasL Tg mice using a similar transgene construct, but detailed histologic and molecular analyses of hearts from their animals were not presented. In addition, FasL mRNA transcripts were determined by RT-PCR, and protein expression was presented for a single line of mice without a positive control, making it impossible to compare the data sets (32). It is unclear why they observed transgene expression in the kidneys of their Tg animals, because the $\alpha-\mathrm{MyHC}$ promoter drives expression specifically in the heart (no transgene expression was detected in the kidneys of our animals).

Our results bear on the controversy over enforced FasL expression. Enforced FasL expression appears to be immune protective in hepatic (20), renal (19), Sertoli cell (11), and myoblast (16) transplantation, and in animal models of arthritis (17) and vascular injury (18). Paradoxically, induced expression of FasL in tumor cells $(21,22)$, islet cells (23), and myoblasts (24) can cause severe neutrophil infiltration and tissue destruction. It has been suggested that coexpression of

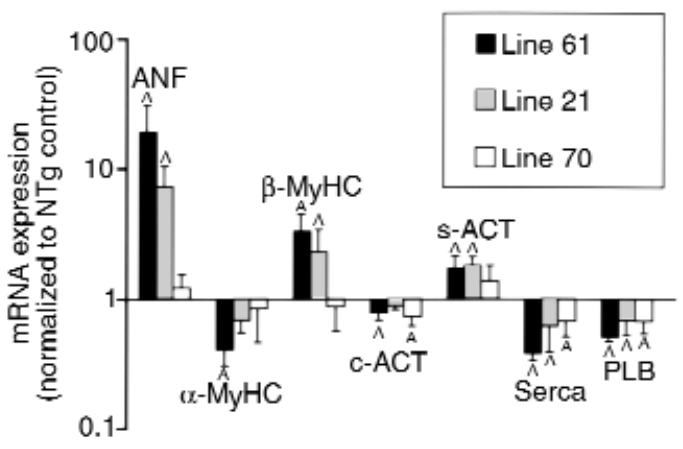

Figure 8

Analysis for cardiac gene expression involved in hypertrophic/failure responses in NTg and FasL Tg hearts. Expression levels for each of the $\mathrm{Tg}$ lines are displayed as mRNA expression relative to NTg expression (baseline $=1.0$ ), with values above and below 1.0 representing increased and decreased expression, respectively (log scale). Six NTg and six Tg hearts from mice 8-12 weeks old were analyzed; all hybridization signals were normalized for loading with GAPDH. FasL Tg lines 21 and 61 (which had the highest copy numbers) demonstrated the changes in cardiac gene expression that are characteristic of hypertrophy ( ${ }^{A} P<$ $0.05)$, with marked ANF induction and actin/ $\mathrm{MyHC}$ isoform switches (fetal gene program reactivation). All Tg hearts demonstrated downregulation of PLB and Serca 2a in a transgene dose-dependent manner. c-ACT, cardiac actin; s-ACT, skeletal actin. 
Fas and FasL on myocytes may target them for destruction by neutrophils (25); however, the absence of cardiomyocyte apoptosis or necrosis in our FasL Tg hearts indicates that Fas and FasL can be coexpressed in the heart without overt tissue destruction. FasLdirected inflammation without tissue destruction has not previously been reported, but our data support the hypothesis that proinflammatory consequences of FasL may be modulated by cellular or microenvironmental factors (26), including FasL expression levels. Recent evidence indicates that the proinflammatory effects of FasL can be modulated by TGF- $\beta 1$ (26). At concentration levels comparable to those measured in Tg hearts, TGF- $\beta 1$ inhibited in vitro FasL-mediated neutrophil cytotoxicity. Cardiac-specific tissue factors such as TGF- $\beta 1$ may prevent neutrophil-mediated tissue destruction in FasL Tg hearts.

Proinflammatory effects of FasL in the heart are proportional to the magnitude of FasL expression. This is pertinent because FasL expression levels were probably different in many of the conflicting studies touching on enforced FasL expression. In particular, varying FasL expression levels may have complicated interpretation of results from studies using cultured myoblasts $(16,25)$, because the opposing studies used different expression vectors to induce FasL expression. Consistent with our data, the results of Li and colleagues (20) indicated that effects of FasL can be dose dependent. Using a FasL-expressing viral plasmid to induce FasL expression in rat livers, they found that high plasmid doses caused fulminant hepatitis, whereas rat livers transfected with a lower plasmid dose were resistant to allograft rejection. This suggests that immune-protective and proinflammatory consequences of FasL may not be mutually exclusive.

A mechanistic explanation for FasL-directed proinflammation remains to be clarified. Because neutrophil infiltration accompanies other examples of enforced FasL expression, it seems likely that this is mediated directly by membrane-bound or soluble FasL. Soluble FasL, generated through metalloproteinase cleavage, is chemotactic for leukocytes and monocytes $(27,28)$. Because all types of leukocytes can express Fas, interaction between Fas-expressing leukocytes and FasL on cardiomyocytes could induce apoptosis of infiltrating leukocytes.

The hypertrophy and interstitial changes observed in FasL-expressing hearts is also obscure. These changes could result from the actions of downstream cytokines released from infiltrating leukocytes, or could be mediated by FasL directly. A variety of cytokines have been implicated in FasL-initiated inflammatory changes, both proinflammatory (IL-1, IL-6, IL-8, and TNF- $\alpha$ ) (2, $23-25,33)$ and anti-inflammatory (TGF- 11$)$ (26). FasL $\mathrm{Tg}$ hearts were found to have transgene dose-dependent increases in TNF- $\alpha$, IL- $1 \beta$, and IL- 6 , all of which can induce cardiac hypertrophy (34-36). Furthermore, there is compelling evidence that such cytokines are integral to various adaptive responses of the heart $(35,37,38)$. In this light, it is intriguing that Tg mice expressing TNF$\alpha$ specifically in the heart demonstrate a phenotype similar to that of FasL Tg mice $(39,40)$. Alternatively, the hypertrophy and interstitial changes of FasL Tg hearts may instead be mediated directly by FasL. Although direct action by FasL upon cardiac myocytes or nonmyocytes has not been observed previously, FasL is a member of the TNF superfamily and shares significant structural and functional similarities with various cytokines. Furthermore, Fas ligation in various cell types has been shown to activate the JNK/MAP kinase $(41,42)$ and PKC (43) signal-transduction pathways, which both participate in cardiac hypertrophic processes.

In conclusion, the data presented here confirm that FasL expression in the heart is proinflammatory, but in contrast to other examples of FasL-mediated inflammation, the consequences appear to be mild, resulting in hypertrophy and interstitial changes rather than tissue destruction. These observations suggest that FasL expression levels and other tissue-specific microenvironmental factors can modulate the proinflammatory actions of FasL.

\section{Acknowledgments}

We thank Jon Neuman and Karen Yager for their help in generating FasL transgenic mice, Bill Pietra and Ron Gill for valuable discussions, and Janet Smith for aid in manuscript preparation. We thank Jim Gulick, Lisa Murray, Adebola Akanbi, and Ken Hance for excellent technical assistance. This work was supported by National Institutes of Health grants SBIR AI-40394, HL-56370, HL41496, HL-56620, HL-52318, and HL-60546.

\footnotetext{
1. Nagata, S., and Golstein, P. 1995. The Fas death factor. Science. 267:1449-1456.

2. Green, D.R., and Ware, C.F. 1997. Fas-ligand: privilege and peril. Proc. Natl. Acad. Sci. USA. 94:5986-5990.

3. Takahashi, T., et al. 1994. Generalized lymphoproliferative disease in mice, caused by a point mutation in the Fas ligand. Cell. 76:969-976.

4. Lynch, D.H., et al. 1994. The mouse Fas-ligand gene is mutated in gld mice and is part of a TNF family gene cluster. Immunity. 1:131-136.

5. Seino, K., et al. 1997. Contribution of Fas ligand to T cell-mediated hepatic injury in mice. Gastroenterology. 113:1315-1322.

6. Baker, M.B.,Altman, N.H., Podack, E.R, and Levy, R.B. 1996. The role of cell-mediated cytotoxicity in acute GVHD after MHC-matched allogeneic bone marrow transplantation in mice. J. Exp. Med. 183:2645-2656.

7. Yamada, K., et al. 1996. Mouse islet cell lysis mediated by interleukin-1induced Fas. Diabetologia. 39:1306-1312.

8. Itoh, N., et al. 1997. Requirement of Fas for the development of autoimmune diabetes in nonobese diabetic mice. J. Exp. Med. 186:613-618.

9. Chervonsky, A.V., et al. 1997. The role of Fas in autoimmune diabetes. Cell. 89:17-24.

10. Giordano, C., et al. 1997. Potential involvement of Fas and its ligand in the pathogenesis of Hashimoto's thyroiditis. Science. 275:960-963.

11. Bellgrau, D., et al. 1995. A role for CD95 ligand in preventing graft rejection. Nature. 377:630-632.

12. Griffith, T.S., Brunner, T., Fletcher, S.M., Green, D.R., and Ferguson, T.A. 1995. Fas ligand-induced apoptosis as a mechanism of immune privilege. Science. 270:1189-1192.

13. Hahne, M., et al. 1996. Melanoma cell expression of Fas(Apo-1/CD95) ligand: implications for tumor immune escape. Science. 274:1363-1366.

14. O'Connell, J., Bennett, M.W., O'Sullivan, G.C., Collins, J.K., and Shanahan, F. 1997. The Fas counterattack: a molecular mechanism of tumor immune privilege. Mol. Med. 3:294-300.

15. Bennett, M.W., et al. 1998. The Fas counterattack in vivo: apoptotic depletion of tumor-infiltrating lymphocytes associated with Fas ligand expression by human esophageal carcinoma. J. Immunol. 160:5669-5675.

16. Lau, H.T., Yu, M., Fontana, A., and Stoeckert, C.J., Jr. 1996. Prevention of islet allograft rejection with engineered myoblasts expressing FasL in
} 
mice. Science. 273:109-112.

17. Zhang, H., et al. 1997. Amelioration of collagen-induced arthritis by CD95 (Apo-1/Fas)-ligand gene transfer. J. Clin. Invest. 100:1951-1957.

18. Sata, M., et al. 1998. Fas ligand gene transfer to the vessel wall inhibits neointima formation and overrides the adenovirus-mediated $\mathrm{T}$ cell response. Proc. Natl. Acad. Sci. USA. 95:1213-1217.

19. Swenson, K.M., et al. 1998. Fas ligand gene transfer to renal allografts in rats: effects on allograft survival. Transplantation. 65:155-160.

20. Li, X.K., et al. 1998. Prolonged survival of rat liver allografts transfected with Fas ligand-expressing plasmid. Transplantation. 66:1416-1423.

21. Arai, H., Gordon, D., Nabel, E.G., and Nabel, G.J. 1997. Gene transfer of Fas ligand induces tumor regression in vivo. Proc. Natl. Acad. Sci. USA. 94:13862-13867.

22. Seino, K., Kayagaki, N., Okumura, K., and Yagita, H. 1997. Antitumor effect of locally produced CD95 ligand. Nat. Med. 3:165-170.

23. Allison, J., Georgiou, H.M., Strasser, A., and Vaux, D.L. 1997. Transgenic expression of CD95 ligand on islet beta cells induces a granulocytic infiltration but does not confer immune privilege upon islet allografts. Proc. Natl. Acad. Sci. USA. 94:3943-3947.

24. Kang, S.M., et al. 1997. Fas ligand expression in islets of Langerhans does not confer immune privilege and instead targets them for rapid destruction. Nat. Med. 3:738-743.

25. Kang, S.M., et al. 1997. Immune response and myoblasts that express Fas ligand. Science. 278:1322-1324.

26. Chen, J.J., Sun, Y., and Nabel, G.J. 1998. Regulation of the proinflammatory effects of Fas ligand (CD95L). Science. 282:1714-1717.

27. Ottonello, L., Tortolina, G., Amelotti, M., and Dallegri, F. 1999. Soluble Fas ligand is chemotactic for human neutrophilic polymorphonuclear leukocytes. J. Immunol. 162:3601-3606.

28. Seino, K., et al. 1998. Chemotactic activity of soluble Fas ligand against phagocytes. J. Immunol. 161:4484-4488.

29. Subramaniam, A., et al. 1991. Tissue-specific regulation of the alphamyosin heavy chain gene promoter in transgenic mice. J. Biol. Chem. 266:24613-24620.

30. Gulick, J., et al. 1997. Transgenic remodeling of the regulatory myosin light chains in the mammalian heart. Circ. Res. 80:655-664.
31. Gerdes, A.M., Onodera, T., Wang, X., and McCune, S.A. 1996. Myocyte remodeling during the progression to failure in rats with hypertension. Hypertension. 28:609-614.

32. Takeuchi, T., et al. 1999. Accelerated rejection of Fas ligand-expressing heart grafts. J. Immunol. 162:518-522.

33. Sata, M., and Walsh, K. 1998. TNFalpha regulation of Fas ligand expression on the vascular endothelium modulates leukocyte extravasation. Nat. Med. 4:415-420.

34. Yokoyama, T., et al. 1997. Tumor necrosis factor-alpha provokes a hypertrophic growth response in adult cardiac myocytes. Circulation. 95:1247-1252

35. Mann, D.L., and Young, J.B. 1994. Basic mechanisms in congestive heart failure. Recognizing the role of proinflammatory cytokines. Chest. 105:897-904.

36. Palmer, J.N., Hartogensis, W.E., Patten, M., Fortuin, F.D., and Long, C.S. 1995. Interleukin-1 beta induces cardiac myocyte growth but inhibits cardiac fibroblast proliferation in culture. J. Clin. Invest. 95:2555-2564.

37. Bozkurt, B., et al. 1998. Pathophysiologically relevant concentrations of tumor necrosis factor-alpha promote progressive left ventricular dysfunction and remodeling in rats. Circulation. 97:1382-1391.

38. Mann, D.L. 1996. The effect of tumor necrosis factor-alpha on cardiac structure and function: a tale of two cytokines. J. Card. Fail. 2(Suppl.):S165-S172.

39. Kubota, T., et al. 1997. Dilated cardiomyopathy in transgenic mice with cardiac-specific overexpression of tumor necrosis factor-alpha. Circ. Res. 81:627-635.

40. Bryant, D., et al. 1998. Cardiac failure in transgenic mice with myocardial expression of tumor necrosis factor-alpha. Circulation. 97:1375-1381.

41. Wilson, D.J., et al. 1996. JNK, but not MAPK, activation is associated with Fas-mediated apoptosis in human $\mathrm{T}$ cells. Eur. J. Immunol. 26:989-994.

42. Brenner, B., et al. 1997. Fas- or ceramide-induced apoptosis is mediated by a Rac1-regulated activation of Jun $\mathrm{N}$-terminal kinase/p38 kinases and GADD153. J. Biol. Chem. 272:22173-22181.

43. Chen, C.Y., and Faller, D.V. 1999. Selective inhibition of protein kinase C isozymes by Fas ligation. J. Biol. Chem. 274:15320-15328. 